\title{
DIFFERENTIABLY SIMPLE ALGEBRAS
}

BY RICHARD E. BLOCK ${ }^{1}$

Communicated by G. D. Mostow, June 3, 1968

In this note we announce a result which gives a complete determination of the differentiably simple algebras (in terms of the simple algebras), together with some related results and applications. At the present stage of the theory the algebras considered are for the most part assumed to be finite-dimensional, but otherwise are completely arbitrary (unless expressly stated), i.e. not necessarily associative and not necessarily having a unit element. The main result is new even in the associative case, solves a conjecture of very long standing in the Lie case, and also leads to the solution of one of the principal problems in the theory of power-associative algebras.

Let $A$ be an algebra over a field $F$. If $D$ is a set of derivations of $A$ (linear transformations $d$ of $A$ into $A$ such that $d(a b)=(d a) b+a(d b)$ for all $a, b$ in $A$ ) then by a $D$-ideal of $A$ is meant an ideal of $A$ invariant under $D ; A$ is called $D$-simple if $A^{2} \neq 0$ and if $A$ has no proper $D$-ideals. Also $A$ is called differentiably simple if it is $D$-simple for some $D$, and hence for the set of all derivations of $A$.

The concept of $D$-simplicity is particularly important at characteristic $p$, where there are $D$-simple algebras which are not simple. Jacobson (see [7]) noted that if $F$ has characteristic $p$, if $S$ is a simple algebra over $F$ and if $G \neq 1$ is a finite elementary abelian $p$-group (so $G$ is the direct product of $n$ copies of the cyclic group of order $p$ ) then the group ring $S G$ (of $G$ with coefficients in $S$ ) is differentiably simple but not simple (and is associative or Lie etc. according as $S$ is); $S G \cong S \otimes{ }_{F} B_{n}(F)$, where $B_{n}(F)$ denotes the (commutative associative) truncated polynomial algebra $F\left[X_{1}, \cdots, X_{n}\right] /\left(X_{1}^{p}, \cdots, X_{n}^{p}\right)$. $\left(B_{n}(F)\right.$ plays an important role, related to the present result, in the theories of simple Lie algebras and certain other algebras at characteristic p.)

TheOREM 1 (MAIN Theorem). If $A$ is a finite-dimensional differentiably simple algebra over a field $F$ then either $A$ is simple or the characteristic is $p$ and there are a simple algebra $S$ over $F$ and an elementary abelian p-group $G \neq 1$ such that $A=S G$.

${ }^{1}$ This work was supported by the National Science Foundation under grants GP 5949 through the University of Illinois and GP 6558 through Yale University. 
As expected from the conclusion the main difficulties in the proof arise at characteristic $p$. In fact in the Lie and associative cases at characteristic 0 the conclusion follows from the old results of Zassenhaus [8] and Hochschild [3] that the radical is a $D$-ideal, and in the characteristic 0 associative case the result is also a special case of a theorem of Posner [4]. At characteristic $p$ the determination of the differentiably simple algebras was considered by Albert [1] and Harper [2], who when $A$ is commutative associative with 1 over an algebraically closed field obtained the result above (i.e. $A \cong B_{n}(F)$ ), and in the Lie case by Zassenhaus [8] and Seligman [7]. In particular Zassenhaus $\left[8\right.$, p. 80] conjectured the result in an equivalent form ${ }^{2}$ (this was the second of three conjectures stated there; the first, the Schreier problem for the outer derivation algebra, remains unsolved, and the third, as noted below, is false). ${ }^{8}$

We recall that a derivation $d$ of $B_{n}(F)$ is determined by the images, which may be arbitrarily chosen elements of $B_{n}(F)$, of the generators $x_{i}=X_{i}+\left(X_{1}^{p}, \cdots, X_{n}^{p}\right)$, so that $d$ may be written $d=\sum_{i=1}^{n} a_{i} \partial / \partial x_{i}$ where $a_{i}=d x_{i}$. Albert [1] showed that $B_{n}(F)$ is not only differentiably simple but that there is even a single derivation $d^{\prime}$ (namely, $\left.\sum_{i=1}^{n}\left(x_{1} \cdots x_{i-1}\right)^{p-1} \partial / \partial x_{i}\right)$ such that $B_{n}(F)$ is $\left\{d^{\prime}\right\}$-simple. It follows that $S \otimes B_{n}(F)$ is $\{d\}$-simple, where $d$ is the derivation defined by $d(s \otimes b)=s \otimes d^{\prime} b$. We have the following stronger result, which is used in the proof of Theorem 1 in showing the existence of Wedderburn decompositions (and thus getting the simple subalgebra $S \otimes 1$ ). Note that if $d$ is a derivation of $A$ and $c \in C$, the centroid of $A$, then $c d$ is a derivation of $A$.

Theorem 2. Suppose $D$ is a set of derivations of a finite-dimensional algebra $A, A$ is $D$-simple, and $D$ is closed under addition, commutation, and left multiplication by all elements of the centroid of $A$. Then $A$ is $\{d\}$-simple for some $d$ in $D$.

In view of Theorem 1 , it is desirable to determine the derivation algebra der $A$ of $A=S \otimes B_{n}(F)$ and to have conditions on a set $D$ of derivations in order for $A$ to be $D$-simple.

2 Zassenhaus conjectured that the differentiably simple Lie algebras are the "power-rings" $S^{(m)}$ which he constructed, with $m=p^{n}$ for some $n$ and $S$ simple. But it can be shown directly that $S^{(m)} \cong S \otimes B_{n}(F)$.

${ }^{3}$ Added September 17, 1968. Sagle and Winter [9] have recently given a short proof of the (comparatively easy) characteristic 0 case of Theorem 1 , by a strictly finitedimensional method. The author has now proved the result of Theorem 1 for $A$ a differentiably simple ring with a minimal (two-sided) ideal. 
Theorem 3. Let $A=S \otimes_{F} B$ where $S$ and $B$ are algebras over a field $F$, $B$ is commutative associative with $1, S^{2}=S$ or $\{s \in S \mid s S=S s=0\}=0$, and $B$ or $S$ is finite-dimensional. Then $\operatorname{der} A=(\operatorname{der} S) \otimes B+\Gamma(S)$ $\otimes \operatorname{der} B$, a direct sum as vector spaces, where $\Gamma(S)$ denotes the centroid of $S$ and

$$
\left(d^{\prime} \otimes b_{1}+\gamma \otimes d^{\prime \prime}\right)(s \otimes b)=d^{\prime} s \otimes b_{1} b+\gamma s \otimes d^{\prime \prime} b .
$$

The answer is a little more complicated if both $S$ and $B$ are infinitedimensional. In the situation of Theorem 3 , if $\Gamma(S)=F$ and if $d$ $=\sum_{i} d_{i}^{\prime} \otimes b_{i}+1_{S} \otimes d^{\prime \prime}$, we say that $d^{\prime \prime}$ is the component of $d$ in $\operatorname{der} B$, and denote it by $d_{B}$.

Proposition 4. Let $A=S \otimes_{F} B_{n}(F)$ where $S$ is central simple and let $D$ be a set of derivations of $A$. Then $A$ is $D$-simple if and only if $B$ $=B_{n}(F)$ is $D_{B}$-simple, where $D_{B}=\left\{d_{B} \mid d \in D\right\}$.

We next consider $D$-semisimplicity and a major application to Lie algebras of characteristic $p$. If $A$ is an algebra and $D$ is a set of derivations of $A$, the sum of all $D$-closed radical ideals is a $D$-closed radical ideal, which we call the $D$-radical. We say that $A$ is $D$-semisimple if its $D$-radical is 0 . For associative algebras we get a nice $D$-structure theory which generalizes the classical Wedderburn Theorem.

Proposition 5. If $A$ is an associative algebra with a set $D$ of derivations and if $M$ is a minimal $D$-ideal of $A$ then either $M^{2}=0$ or $M$ is $(D \mid M)$-simple. If in addition $A$ is finite-dimensional (over a field) and $D$-semisimple then $A$ is a direct sum of all of its minimal D-ideals (and hence of algebras which are either simple or of the form $S G$ ).

A similar result (with a more complicated proof) holds for those classes of power-associative algebras in which ideals of semisimple algebras have a unit element. However, the result is false for Lie algebras. Note that in a Lie algebra $L$ a nonabelian ideal $M$ is minimal if and only if $M$ is $\left(\operatorname{ad}_{M} L\right)$-simple. This has motivated the study of differentiably simple Lie algebras.

Proposition 6. Let $D$ be a set of derivations of a finite-dimensional Lie algebra $L$ and suppose that $L$ is $D$-semisimple. Then $L$ has only finitely many minimal $D$-ideals, say $L_{1}, \cdots, L_{n}$, their sum $M$ is direct, each $L_{i}$ is $\left(D \mid L_{i}\right) \cup_{\operatorname{ad}_{i}} L$-simple, $x \rightarrow \operatorname{ad}_{M} x$ is an isomorphism of $L$ onto a subalgebra of der $M$ containing inder $M$, and the mapping $d \leftrightarrow d \mid M$ of $D$ into der $M$ is one-one.

With $D=\operatorname{der} L$ this gives the results of Seligman [7] in a sharper form (in this case one also uses the terms characteristic ideal and 
characteristically simple (semisimple)). We call $M$ the $D$-socle of $L$. If $L$ is semisimple this equals the socle of $L$ (the sum of the minimal ideals).

The above results lead to a description of all the semisimple Lie algebras in terms of the socle. Let $S_{1}, \cdots, S_{r}$ be central simple Lie algebras over a field $F$ of characteristic $p$, and let $n_{1}, \cdots, n_{r}$ be nonnegative integers (not necessarily distinct). We write $S$ $=\oplus \sum_{i=1}^{r} S_{i} \otimes B_{i}$ where $B_{i}$ denotes $B_{n_{i}}(F)$ and $B_{i}=F$ if $n_{i}=0$, and identify $S$ with inder $S$, so that

$$
\begin{aligned}
S & =\operatorname{inder} S=\oplus \sum_{i=1}^{r}\left(\operatorname{inder} S_{i}\right) \otimes B_{i} \subseteq \operatorname{der} S \\
& =\oplus \sum_{i=1}^{r}\left(\left(\operatorname{der} S_{i}\right) \otimes B_{i}+1_{S_{i}} \otimes \operatorname{der} B_{i}\right) .
\end{aligned}
$$

TheOREM 7. Let $L$ be any subalgebra of $\operatorname{der} S$ containing $S$ (hence $L$ is uniquely determined by a subalgebra of $\oplus \sum_{i=1}^{r}$ ((outder $\left.S_{i}\right) \otimes B_{i}+1_{S_{i}}$ $\left.\left.\otimes \operatorname{der} B_{i}\right)\right)$. Then $L$ is semisimple if and only if $B_{i}$ is $L_{B_{i}}$-simple for $i=1, \cdots, r$, where $L_{B_{i}}$ is the set of components of elements of $L$ in $\operatorname{der} B_{i}$. If $F$ is algebraically closed then every finite-dimensional semisimple Lie algebra over $F$ has this form, and the semisimple algebra uniquely determines the pairs $\left(S_{i}, n_{i}\right)$ up to isomorphism and reordering. For a semisimple $L$ the mapping $x \mapsto \operatorname{ad}_{L} x\left(x \in N_{\mathrm{der}}{ }_{S} L\right)$ is an isomorphism of the normalizer of $L$ in der $S$ onto der $L$. The same results hold with differentiably (=characteristically) semisimple in place of semisimple provided the condition on $L_{B_{i}}$ is replaced by the same condition on $\left(N_{\text {der } s} L\right)_{B_{i}}$.

Let $F$ be an algebraically closed field of characteristic $p>3$. There is a conjecture [8], [5] that perfect semisimple Lie algebras over $F$ are direct sums of simple algebras. This would imply the Schreier conjecture on outer derivations, but it is false. In fact taking $r=1$, $S_{1}$ a simple algebra with all derivations inner, $n_{1}>0$, and $L=\operatorname{der} S$ above, we get a semisimple algebra with unique ideal $S$ and with $L / S \cong W_{n_{1}}$, the Witt algebra. What is true is that every simple Lie algebra over $F$ has its outer derivation algebra solvable if and only if every perfect semisimple Lie algebra over $F$ with all minimal ideals simple is a direct sum of simple algebras. Kostrikin [6] has proved if $p>5$ that semisimple Lie algebras of dimension $<p$ over $F$ are direct sums of simple algebras (of classical type), and Theorem 7 gives a generalization of this.

The application of Theorem 1 to power-associative algebras, giving 
a uniform determination of $A^{+}$for flexible simple algebras $A$ (including the previously unsettled degree two case), will be announced in another article.

\section{REFERENCES}

1. A. A. Albert, On commutative power-associative algebras of degree two, Trans. Amer. Math. Soc. 74 (1953), 323-343.

2. L. R. Harper, Jr., On differentiably simple algebras, Trans. Amer. Math. Soc. 100 (1961), 63-72.

3. G. Hochschild, Semi-simple algebras and generalized derivations, Amer. J. Math. 64 (1942), 677-694.

4. E. C. Posner, Differentiably simple rings, Proc. Amer. Math. Soc. 11 (1960), 337-343.

5. I. Kaplansky, "Lie algebras" in Lectures on modern mathematics, Vol. I, edited by T. L. Saaty, Wiley, New York, 1963, Chapter 5.

6. A. I. Kostrikin, Squares of adjoint endomorphisms in simple Lie p-algebras, Izv. Akad. Nauk SSSR Ser. Mat. 31 (1967), 445-487. (Russian)

7. G. B. Seligman, Characteristic ideals and the structure of Lie algebras, Proc. Amer. Math. Soc. 8 (1957), 159-164.

8. H. Zassenhaus, Über Lie'sche Ringe mit Primzahlcharakteristik, Abh. Math. Sem. Univ. Hamburg 13 (1939), 1-100.

9. A. Sagle and D. J. Winter, On homogeneous spaces and reductive subalgebras of simple Lie algebras, Trans. Amer. Math. Soc. 128 (1967), 142-147.

UNIVERSITY OF ILLINOIS 